\title{
PIGF-MMP9-engineered iPS cells supported on a PEG-fibrinogen hydrogel scaffold possess an enhanced capacity to repair damaged myocardium
}

\author{
C Bearzi ${ }^{1,2}$, C Gargioli ${ }^{1}$, D Baci ${ }^{1}$, O Fortunato ${ }^{1}$, K Shapira-Schweitzer ${ }^{3}$, O Kossover $^{3}$, MV G Latronico ${ }^{4}$, D Seliktar ${ }^{3}$, G Condorelli $^{4}$ \\ and R Rizzi ${ }^{\star, 1,2}$
}

Cell-based regenerative therapies are significantly improved by engineering allografts to express factors that increase vascularization and engraftment, such as placental growth factor (PIGF) and matrix metalloproteinase 9 (MMP9). Moreover, the seeding of therapeutic cells onto a suitable scaffold is of utmost importance for tissue regeneration. On these premises, we sought to assess the reparative potential of induced pluripotent stem (iPS) cells bioengineered to secrete PIGF or MMP9 and delivered to infarcted myocardium upon a poly(ethylene glycol)-fibrinogen scaffold. When assessing optimal stiffness of the PEG-fibrinogen (PF) scaffold, we found that the appearance of contracting cells after cardiogenic induction was accelerated on the support designed with an intermediate stiffness. Revascularization and hemodynamic parameters of infarcted mouse heart were significantly improved by injection into the infarct of this optimized PF scaffold seeded with both MiPS (iPS cells engineered to secrete MMP9) and PiPS (iPS cells engineered to secrete PIGF) cells as compared with nonengineered cells or PF alone. Importantly, allograft-derived cells and host myocardium were functionally integrated. Therefore, survival and integration of allografts in the ischemic heart can be significantly improved with the use of therapeutic cells bioengineered to secrete MMP9 and PIGF and encapsulated within an injectable PF hydrogel having an optimized stiffness.

Cell Death and Disease (2014) 5, e1053; doi:10.1038/cddis.2014.12; published online 13 February 2014

Subject Category: Experimental Medicine

The use of cell-based protocols for regenerative medicine in cardiology is hindered by the complexity of obtaining a reliable source of functional cardiomyocytes $(\mathrm{CMs})^{1}$ and by the low survival rate of allografts within recipient myocardium. ${ }^{2}$ Regarding the first point, induced pluripotent stem (iPS) cells are a very promising source of stem cells that are relatively easily generated by the reprogramming of adult somatic cells, such as fibroblasts, and that can be differentiated into all cell types of the body, including CMs. ${ }^{3,4}$ We have previously reported that iPS cells can be generated - through the conventional 'Yamanaka approach' - from still-replicating 1-day-old mouse CMs, and that these cells more efficiently differentiate into cardiac progenitors than iPS cells generated from other cell types: in fact, the efficiency of cardiogenic differentiation of CM-derived iPS cells was $53-57 \%$ compared with $33-39 \%$ for mouse embryonic stem cells and $25-33 \%$ for cardiac fibroblast-derived iPS cells. ${ }^{5}$ However, how stem cells adapt to a hostile environment, such as the postinfarct myocardium, and whether they efficiently differentiate after engraftment toward the $\mathrm{CM}$ lineage, overcoming survival stresses, is not known. ${ }^{6}$
On the second point, survival of engrafted cells within damaged myocardial tissue is critically dependent upon support by an adequate blood supply. We have demonstrated that the survival of cell allografts within muscle can be improved by bioengineering them to express specific factors: in fact, when engrafted in a model of muscle dystrophy, mesangioblast stem cells forced to secrete placental growth factor (PIGF) $)^{7,8}$ and matrix metalloproteinase 9 (MMP9) $^{9}$ produced significant improvements in muscle performance. ${ }^{10}$ PIGF is a well-known angiogenic factor that enhances neoangiogenesis and that attracts host vessels. ${ }^{11}$ It has a higher disease-specific activity than other angiogenic factors, such as vascular endothelial growth factor (VEGF) and other cytokines, and therefore does not affect quiescent vessels in healthy tissues. As a consequence, PIGF is an attractive therapeutic tool for treating ischemic diseases. ${ }^{12}$ Moreover, in the context of acute myocardial infarction (AMI), the generation of scar tissue hinders the revascularization process. Secretion by the allograft of MMP9 - an enzyme that breaks down scar tissue and, thus, permeabilizes the extracellular matrix (ECM), remodeling tissues in pathological

\footnotetext{
${ }^{1}$ Istituto Ricovero Cura Carattere Scientifico MultiMedica, Milan, Italy; ${ }^{2}$ Cell Biology and Neurobiology Institute, National Research Council of Italy (CNR), Rome, Italy; ${ }^{3}$ Faculty of Biomedical Engineering, Technion-Israel Institute of Technology, Haifa, Israel and ${ }^{4}$ Humanitas Clinical and Research Center, Rozzano, Milan, Italy ${ }^{*}$ Corresponding author: R Rizzi, Institute of Cell Biology and Neurobiology, Consiglio Nazionale delle Ricerche, Via Fantoli 16/15, MultiMedica IRCCS, Via Fantoli 16/15, 20137 Milan, Italy. Tel: +39 0250995 663; Fax: +39 0250995 661; E-mail roberto_rizzi@ hotmail.com

Keywords: cardiac tissue engineering; hydrogel; induced pluripotent stem cells; angiogenesis; matrix metalloproteinase

Abbreviations: AMI, acute myocardial infarction; BMP2, bone morphogenetic protein 2; CM, cardiomyocyte; cTNNI, cardiac troponin I; EB, embryoid body; ECM, extracellular matrix; FS, fractional shortening; HUVEC, human umbilical vein endothelial cell; iPS cells, induced pluripotent stem cell; VTI, velocity/time integral; MEF, mouse embryonic fibroblast; MiPS cells, iPS cells engineered to secrete MMP9; MMP9, matrix metalloproteinase 9; PEG-DA, poly(ethylene glycol)-diacrylate; PF, PEG-fibrinogen; PiPS cells, iPS cells engineered to secrete PIGF; PIGF, placenta growth factor; SCF, stem cell factor

Received 07.11.13; revised 06.12.13; accepted 12.12.13; Edited by G Melino
} 
processes $^{9,13}$ - overcomes this limitation by creating an environment conducive to the migration of resident and/or circulating stem cells. ${ }^{13}$

Another fundamental aspect that improves the survival of cell allografts and, hence, the repair of damaged tissue, is the presence of a biomimetic scaffold onto which the therapeutic cells can be seeded. Biomaterial scaffolds can protect transplanted cells, preventing cell death by improving proliferation and/or reducing apoptosis. Different types of biomaterials constructed from polymers or copolymers of natural or synthetic origin have been used for engrafting cells in the myocardium. ${ }^{14,15}$ Among these, hydrogels are being increasingly used to mimic the natural ECM in threedimensional (3D) scaffolds. ${ }^{16,17}$ A semisynthetic hydrogel composed of a fibrinogen backbone crosslinked with bifunctional poly(ethylene glycol) (PEG) side chains was previously used by our group for cardiac and skeletal muscle regeneration. The PEG-fibrinogen (PF) hydrogel was used to make 3D cellularized hydrogel constructs by seeding cells into a liquid solution of PF containing a photoinitiator. Exposure to longwavelength UV light initiates polymerization of the scaffold. This type of hydrogel has a distinct advantage over other types of scaffold because its mechanical properties are highly malleable while leaving the functionality of the encapsulated cells well preserved by the backbone of the polymeric network. In fact, the biological domains in the fibrinogen backbone provide attachment motifs for endothelial and smooth muscle cell adhesion necessary for the development of a blood supply and of newly formed tissue, as well as proteolytic sensitivity to cell-mediated biodegradation. ${ }^{16,17}$ Moreover, the shear storage modulus $\left(G^{\prime}\right)$ of the PF scaffold which is dependent upon the molecular weight of the PEG constituent, and is proportional to the percent polymeric composition and crosslinking - can be altered to suit the characteristics of the recipient tissue and the donor cells.

Thus, a feasible strategy to overcome poor allograft survival in cardiac muscle reconstruction would consist of a combinatorial approach comprising the bioengineering of therapeutic cells so that they express factors that facilitate vascularization, and the delivery of these cells on a support optimized for the damaged myocardium. For the present report, we employed iPS cells generated from 1-day-old mouse CM because they represent an efficient model with which to study myocardial regeneration - that we transduced with lentiviral vectors encoding MMP9 or PIGF. We then assessed the capacity of these bioengineered iPS cells to aid myocardial repair in an AMI mouse model, by injecting them into infarcts as part of a cell-seeded PF hydrogel construct.

\section{Results}

Characterization of bioengineered iPS cells. After ascertaining that bioengineered iPS cells were capable of synthesizing PIGF and MMP9 (Figure 1a and Supplementary Figure 1 online), we examined the biological activity of the secreted factors. For MMP9, we used a cell invasion assay to measure the migratory capacity of iPS cells stimulated by a chemoattractant, either stromal cell-derived factor-1 (SDF-1) or stem cell factor (SCF): MMP9-secreting iPS (MiPS) cells were significantly better able to digest the matrigel barrier and migrate into the lower compartment of a Boyden chamber than control iPS cells (Figures $1 \mathrm{~b}$ and $\mathrm{c}$ ). The biological activity of PIGF was assessed by analyzing the number and the length of capillary-like structures formed by human umbilical vein endothelial cells (HUVECs) exposed to conditioned media: HUVECs grown in a conditioned medium from PIGF-secreting iPS (PiPS) cells had a significantly enhanced angiogenic capacity with respect to those kept in medium originating from control iPS cell cultures (Figures 1d and e).

In vitro biocompatibility of iPS cell-scaffold constructs. We then assessed the effect of culturing iPS cells with the PF scaffold using the matrix stiffness to optimize either survival or cardiac differentiation. The iPS cells - as for embryonic stem cells - must be cultured on a mouse embryonic fibroblast (MEF) feeder layer to prevent them from differentiating. We examined stiffness-optimized PF scaffolds supporting iPS cell cultures as an alternative to MEF feeder layers. In addition, modulation of PF stiffness was used to optimize 3D cardiac muscle tissue formation in vitro using dispersed encapsulated iPS cells. PEG-diacrylate (PEG-DA) crosslinker was added to the PF in order to increase its stiffness while maintaining iPS cell stemness and/or facilitating cardiac differentiation. ${ }^{18}$ To this end, three different scaffold compositions were examined: PF without any additional crosslinker, a low stiffness $\left(G^{\prime} \sim 120 \mathrm{~Pa}\right)$ composition; PF plus an additional 1\% PEG-DA, an intermediate stiffness $\left(G^{\prime} \sim 350 \mathrm{~Pa}\right)$ composition; and PF plus an additional $2 \%$ PEG-DA, a high stiffness $\left(G^{\prime} \sim 700 \mathrm{~Pa}\right)$ composition. $^{18,19}$

We did not observe any effect of increasing stiffness on iPS cell proliferation: in fact, no morphological differences were discernible in iPS cell colonies cultured on MEF or on either of the three scaffold compositions (Figure 2a). Importantly, iPS cell lines were still capable of growing and maintaining their undifferentiated state when cultured on a PF scaffold in the absence of a feeder layer. Quantitative real-time-PCR (qRT-PCR) demonstrated that the expression of the stem cell markers Oct4, Sox2, and Nanog remained stable and long-lasting when iPS cells were grown on the PF hydrogels, and was comparable to iPS cells cultured on MEF (Figure $2 b$, Supplementary Table 1 online). Culturing on the hydrogel had the additional advantage of increasing cell purity by removing contamination by MEF. Immunofluorescence staining for the embryonic antigen stage-specific embryonic antigen 1 (SSEA1) confirmed stemness maintenance of all iPS cell lines after 14 days of culture on PF supplemented with an additional 1\% PEG-DA (Figure 2c).

We then investigated the cardiac differentiation capacity of dispersed iPS cell lines in 3D culture. To this end, we used a differentiation protocol consisting of a defined concentration of bone morphogenetic protein 2 (BMP2), 5,20 induction of embryoid bodies (EBs), and then encapsulation in PF. We found that EBs cultured in the intermediate-stiffness composition (i.e., PF enriched with an additional 1\% PEG-DA) started beating 1 day earlier than those cultured with the standard protocol - in which EBs are plated on gelatinized dishes - or on the other two scaffold compositions (Supplementary Video 1 online). qRT-PCR revealed that EBs encapsulated in the PF 
a

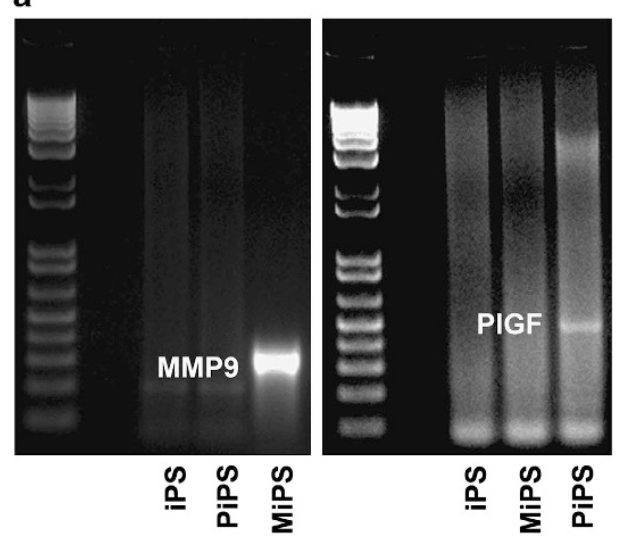

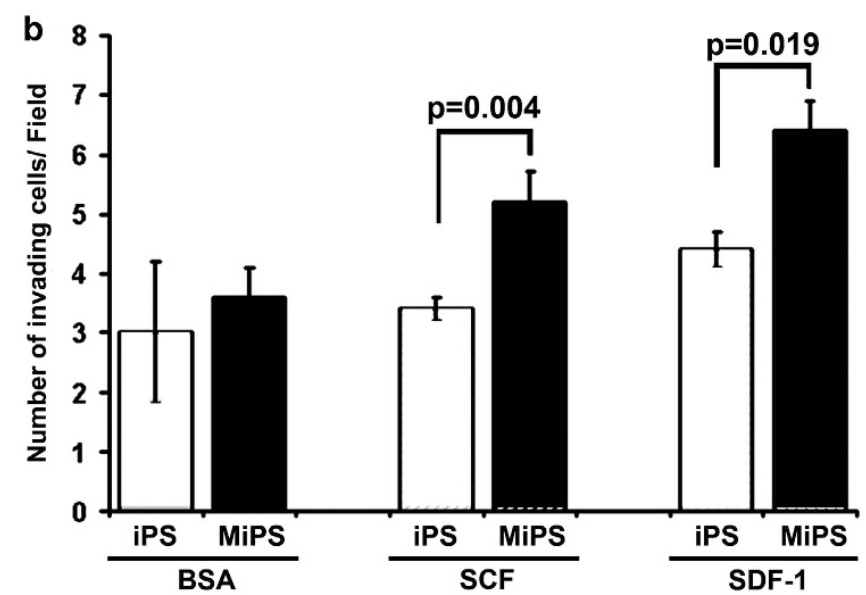

c
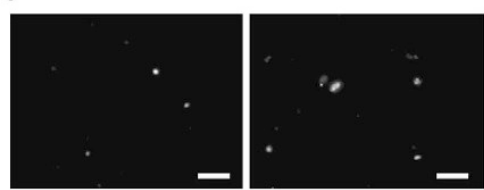

iPS

MiPS

BSA
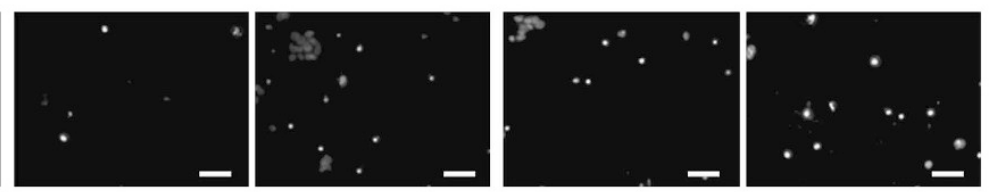

MiPS

SCF

MiPS

d
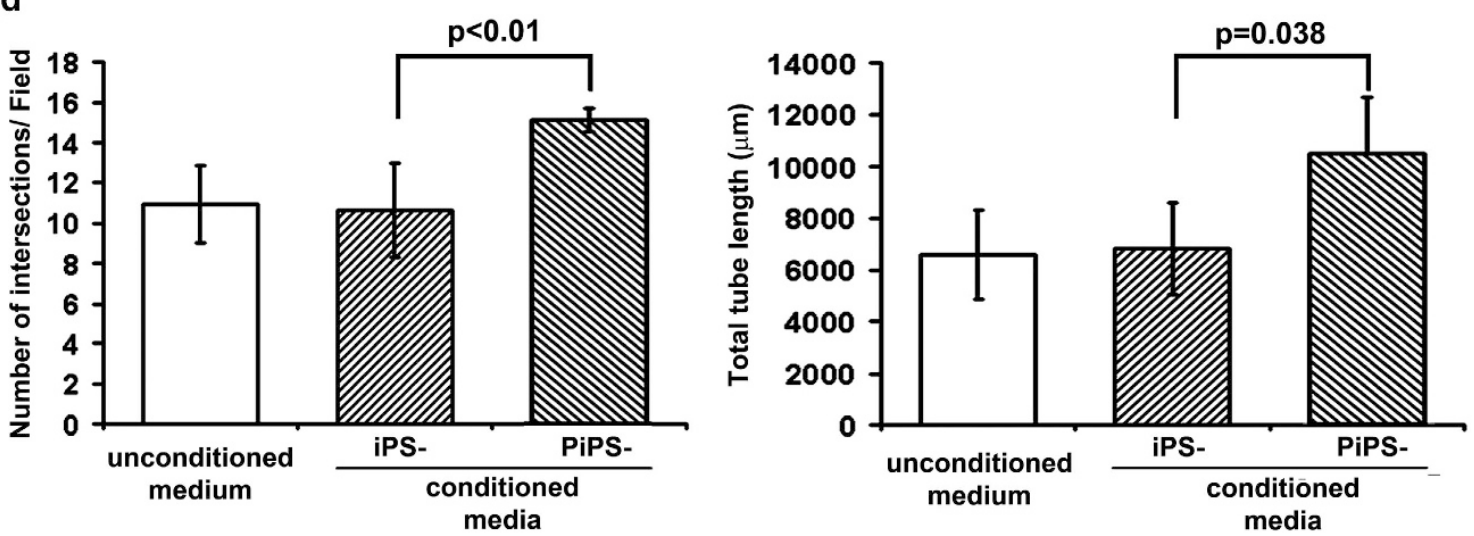

e

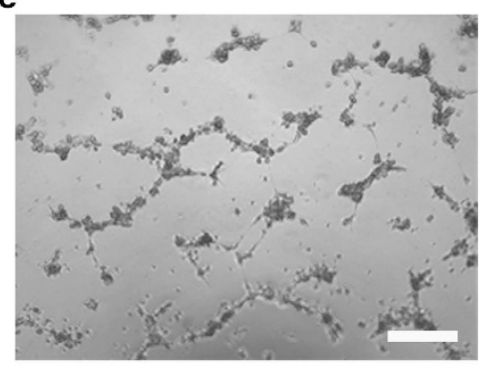

unconditioned medium

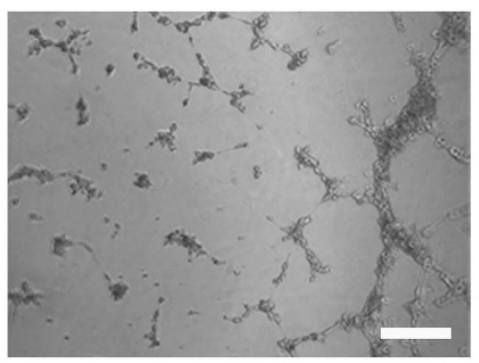

iPS-

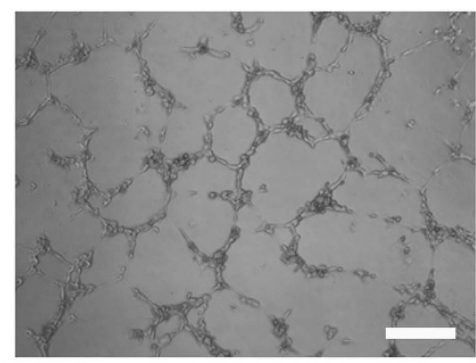

PiPS-

conditioned media

Figure 1 Characterization of iPS cells bioengineered to express MMP9 or PIGF. (a, left) RT-PCR showing the presence of an MMP9 transcript in iPS cells bioengineered to secrete MMP9 (MiPS) into the culture medium; (a, right) RT-PCR illustrating that the PIGF transcript was produced by iPS cells engineered to secrete PIGF (PiPS). iPS cells transduced with an $n L a c Z$ viral vector (iPS) were used as a negative control. (b) Cell invasion experiments attested an enhanced aptitude of MiPS cells, with respect to control iPS cells, to permeabilize the matrix and migrate when attracted by either SCF or SDF-1. The graph gives the number of invading cells/field on the y axis. BSA (bovine serum albumin) was used as the control condition. Mean \pm S.E.M.; $N=3$. (c) Representative images of DAPI-stained nuclei in invasion experiments. iPS and MiPS cells were stimulated to migrate through a matrigel membrane by either SCF or SDF-1. Magnification $\times 20$; white bars $=20 \mu \mathrm{m}$. (d, left) Number of capillary-like structures generated by HUVECs after exposure to conditioned media; (d, right) length of the newly formed capillaries after exposure to conditioned media. Mean \pm S.E.M.; $N=3$. (e) Representative images of capillary-like structures formed by HUVECs after exposure to control iPS- or PiPS cell-conditioned media. Magnification $\times 5$; white bars $=100 \mu \mathrm{m}$ 

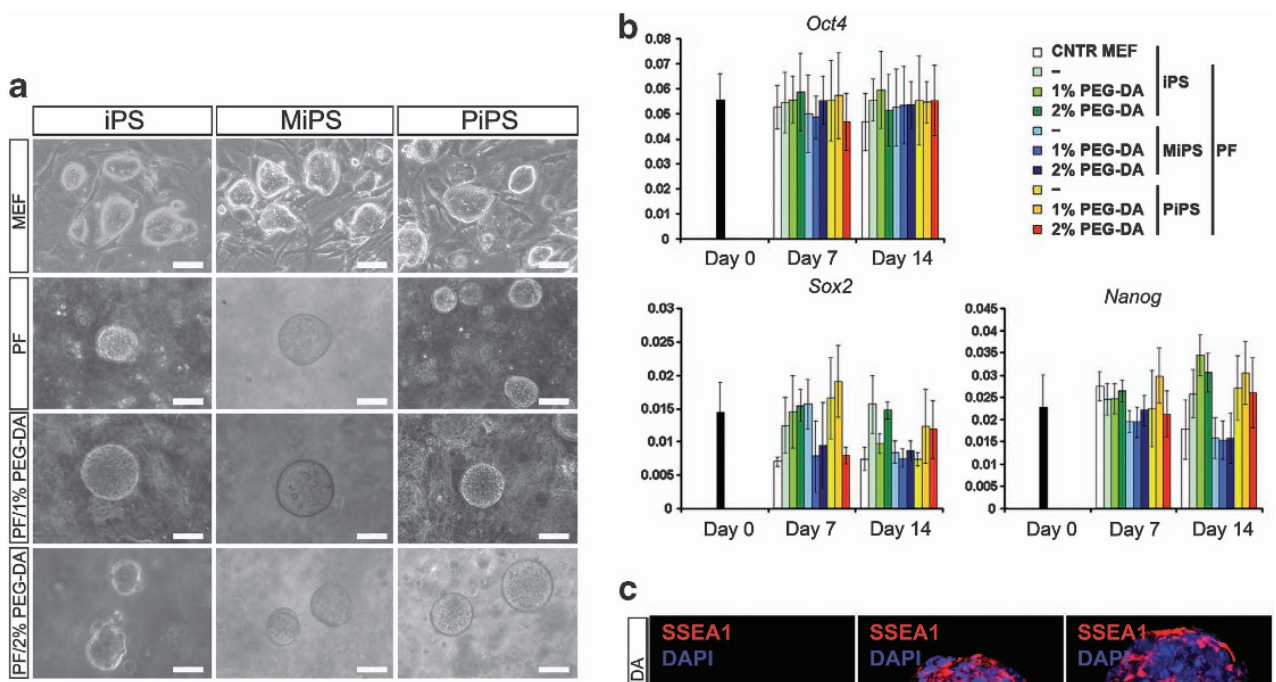

C

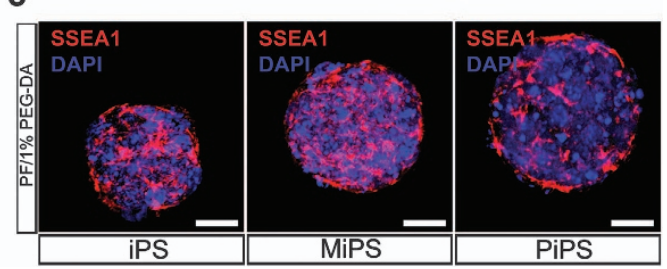

Figure 2 Effect of growing iPS cells on PEG-fibrinogen scaffolds. (a) Morphology of IPS, MiPS, and PiPS cell colonies cultured on mouse embryonic fibroblast (MEF) feeder layers (upper row), on PEG-fibrinogen (PF) scaffolds without a feeder layer (second row), or on PF supplemented with additional (1 and $2 \%$ ) PEG-diacrylate (PEG-DA) in the absence of MEFs (lower two rows). White bars $=100 \mu \mathrm{m}$. (b) qRT-PCR attesting the expression of three embryonic genes after 7 days and 14 days of culture. iPS, MiPS, and PiPS cells maintain their pluripotent status when grown on MEF (CNTR MEF), PF, or 1 and $2 \%$ PEG-DA-enriched PF. Bar graphs express $\Delta \Delta C \mathrm{Ct}$ values. Mean \pm S.E.M.; $N=4$. (c) Immunofluorescence for SSEA1 in iPS, MiPS, and PiPS cells after 14 days of culture on PF supplemented with an additional $1 \%$ PEG-DA. Nuclei were stained with DAPI. White bars $=100 \mu \mathrm{m}$

hydrogel compositions maintained a higher expression of early (Brachyury) and late (Mhy6) cardiac genes (Figure 3a and Supplementary Figure 2 and Supplementary Table 1 online). The long-lasting expression of the genes responsible for the extreme phases of cardiomyogenesis (i.e., Brachyury and Mhy6) led us to speculate that the intermediate-stiffness composition promoted a more stable differentiation process in iPS cells when in 3D culture, and resulted in a higher rate of cell maturation.

We used qRT-PCR to quantitatively define the relationship between the intermediate-stiffness PF scaffold and the increased pattern of $\mathrm{CM}$ differentiation. Surprisingly, we found that encapsulation in this PF hydrogel composition significantly affected genes involved in apoptosis: in fact, the expression of apoptotic genes (caspase $3, \mathrm{Bcl} 2$, and p21) was significantly decreased (Figure $3 \mathrm{c}$ ), whereas proliferation genes (cyclins D1 and E1) were unaffected (Figure 3b and Supplementary Table 1 online). Immunofluorescence assays confirmed the expression of the cardiac antigen $\alpha$-sarcomeric actin ( $\alpha$-Sarc) after 3,5 , and 7 days of differentiation of PiPS cells encapsulated in the intermediate-stiffness scaffold (Figure 3d).

\section{Biocompatibility of iPS cell-scaffold constructs in vivo.}

We have previously demonstrated that with respect to other cell types, iPS cells generated from neonatal CMs have an enhanced efficiently for redifferentiation into CMs. ${ }^{5}$ Because growth and maturation of cells are improved by $3 \mathrm{D}$ culture procedures, we tested the biocompatibility and differentiation capacity of our model in vivo. Accordingly, constructs consisting of $20 \mu \mathrm{l}$ of polymerized intermediate-stiffness scaffold encapsulating either $1 \times 10^{6}$ PiPS or iPS cells at 5 days of differentiation were introduced onto the surface of tibialis anterior (TA) muscle of immunodeficient mice. One month after subcutaneous implantation, any newly formed structures were removed and analyzed with histology and FACS.

Constructs seeded with differentiated PiPS cells generated larger structures with less necrosis than those prepared with differentiated control iPS cells (Figure $4 a$, left panels). Moreover, these structures contained cells that were positive for $\alpha$-Sarc and $\alpha$-smooth muscle actin ( $\alpha$-SMA; Figure $4 a$, middle and right panels). The latter cells formed arteriolar-like structures, indicative of arteriogenesis. The exogenous nature of these cells was attested by positivity for nLacZ. Some of these newly formed structures were removed from the mice and enzymatically digested, and the isolated cells cultured on gelatinized dishes. We observed that cultures contained small foci of beating cells (Supplementary Video 2 online). FACS analysis for cardiac troponin I (cTNNI) and $\beta$-galactosidase $(\beta$-gal) determined that a higher number of LacZ-positive cells were co-positive for cTNNI in structures generated from PiPS cells (Figure 4b). Moreover, a higher vessel density was found in tissue generated from differentiated PiPS cells (Figure 4c).

iPS cell-scaffold constructs in an AMI mouse model. The effect of the implant was then studied in a model of AMI. Female NOD-SCID mice had their left descending coronary artery surgically ligated to produce an ischemic area in the left ventricle. Implants prepared from intermediate-stiffness 
a

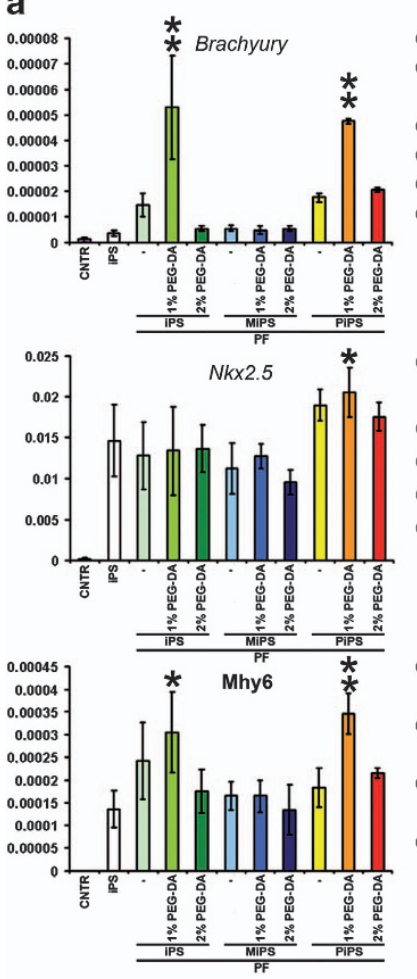

b
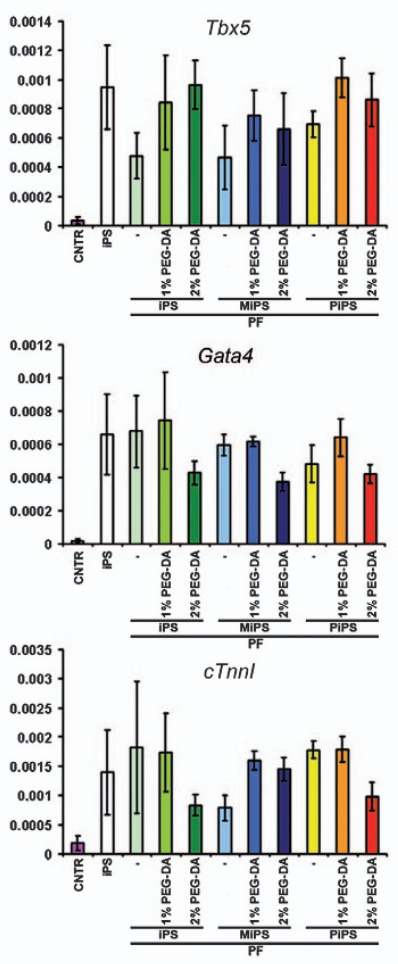

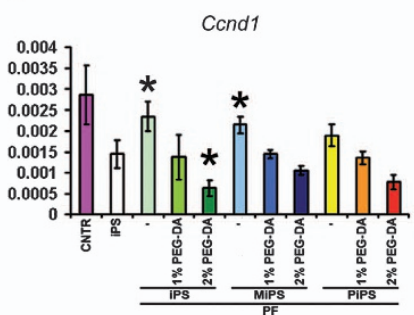

p53

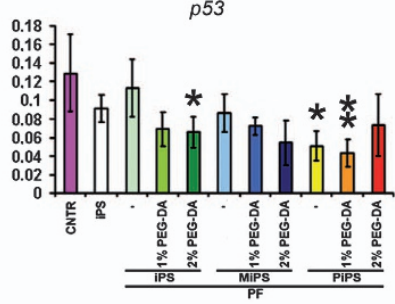

Cone1

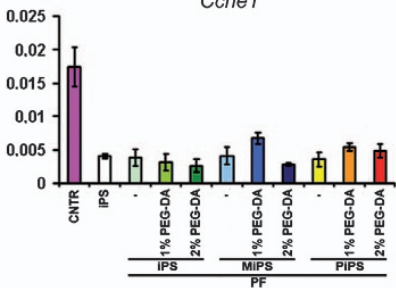

C

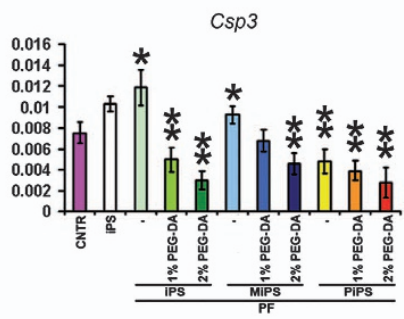

$B c / 2$

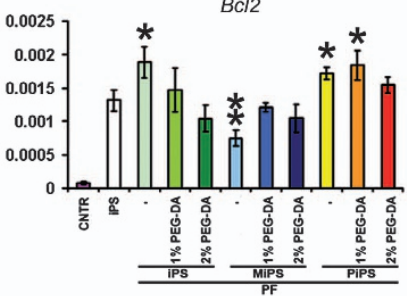

p21

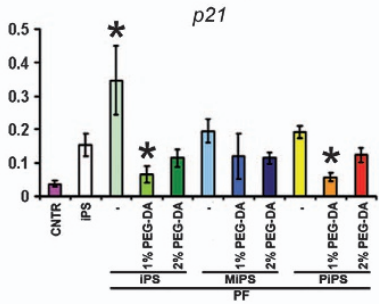

d
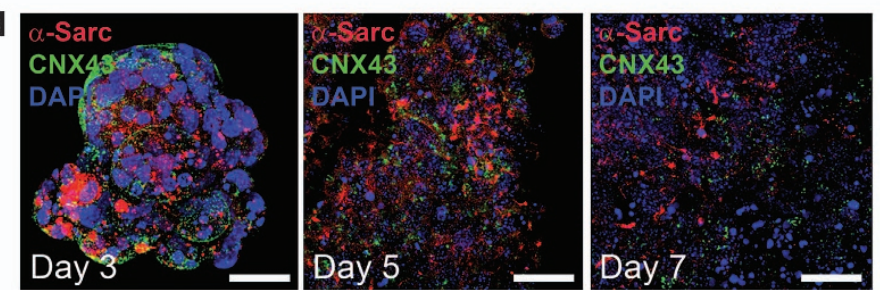

Figure 3 Gene expression in iPS cell lines seeded onto PF scaffolds with differing stiffnesses. (a) qRT-PCR was used to assess expression of early (Brachyury and Tbx5), intermediate (Nkx2.5 and Gata4), and late (Mhy6 and cTnn) cardiac genes 7 days after differentiation. (b) qRT-PCR for the expression of genes related to proliferation (Ccnd1, p53, and Ccne1). (c) qRT-PCR assays for the expression of genes related to apoptosis (Csp3, Bcl2, and p21). In (a-c), controls are represented by undifferentiated iPS cells (CNTR) and by differentiated iPS cells cultured on gelatin (iPS). ${ }^{*} P<0.05,{ }^{* *} P<0.01$ versus iPS cells. Bar graphs express mean $\Delta \Delta$ Ct values \pm S.E.M.; $N=4$ (a-c). (d) Immunofluorescence for the cardiac proteins $\alpha$-sarcomeric actin ( $\alpha$-Sarc, red) and connexin 43 (CNX43, green) after 3,5 , and 7 days of differentiation in PiPS cells seeded onto a PEG-fibrinogen (PF) scaffold stiffened with an additional 1\% PEG-diacrylate (PEG-DA). Nuclei were stained with DAPI (blue). White bars $=200 \mu \mathrm{m}$ (for day 3 ) and $100 \mu \mathrm{m}$ (for days 5 and 7 )

scaffolds seeded with iPS cells generated from male mice were then immediately injected into the ischemic myocardium of the female mice. PF alone was injected either into the border zone or into the infarct to evaluate the supporting functions of the scaffold after the ischemic event.

The presence of allograft cells was ascertained by fluorescent in situ hybridization for the $Y$ chromosome (Figure 5a). Importantly, male-derived iPS cells were able to integrate functionally with the female host tissue. Gapjunction formation - identified as positivity for connexin 43 (CNX43) - was found between allograft and host cells. Moreover, the data suggested that the muscle origin of the grafted iPS cells may have facilitated transdifferentiation into SMA-positive cells that are necessary for the development of a blood supply to the infarcted area.

Histological analysis highlighted an increase in capillary density and angiogenesis, and a decrease in fibrotic and apoptotic indexes, in AMI mice receiving the various iPS cellPF implants as compared with controls (Figure 5b). Apoptosis was also markedly reduced in mice treated only with the scaffold, confirming previous results in this direction.

The mice were also monitored for 30 days to assess hemodynamic parameters. Percent fractional shortening (\%FS) was drastically reduced in the PBS control group 30 days after $\mathrm{AMI}(21 \pm 1 \%)$, whereas mice treated with iPS cells only $(30.3 \pm 1.3 \%)$, scaffold only $(25 \pm 1.1 \%)$, or with the iPS cell-scaffold construct $(32.3 \pm 3.5 \%)$ had relatively slower time-dependent reductions in this parameter. On the other hand, treatments conducted with engineered iPS cells produced a partial recovery of cardiac function (MiPS cellscaffold, $31 \pm 3 \%$; PiPS cell-scaffold, $34 \pm 1 \%$ ), whereas the combined use of MiPS with PiPS cells within the scaffold produced the best therapeutic outcome (37 $\pm 1.8 \%$; Figure $5 \mathrm{c}$ ). The velocity/time integral (VTI) - which reflects 

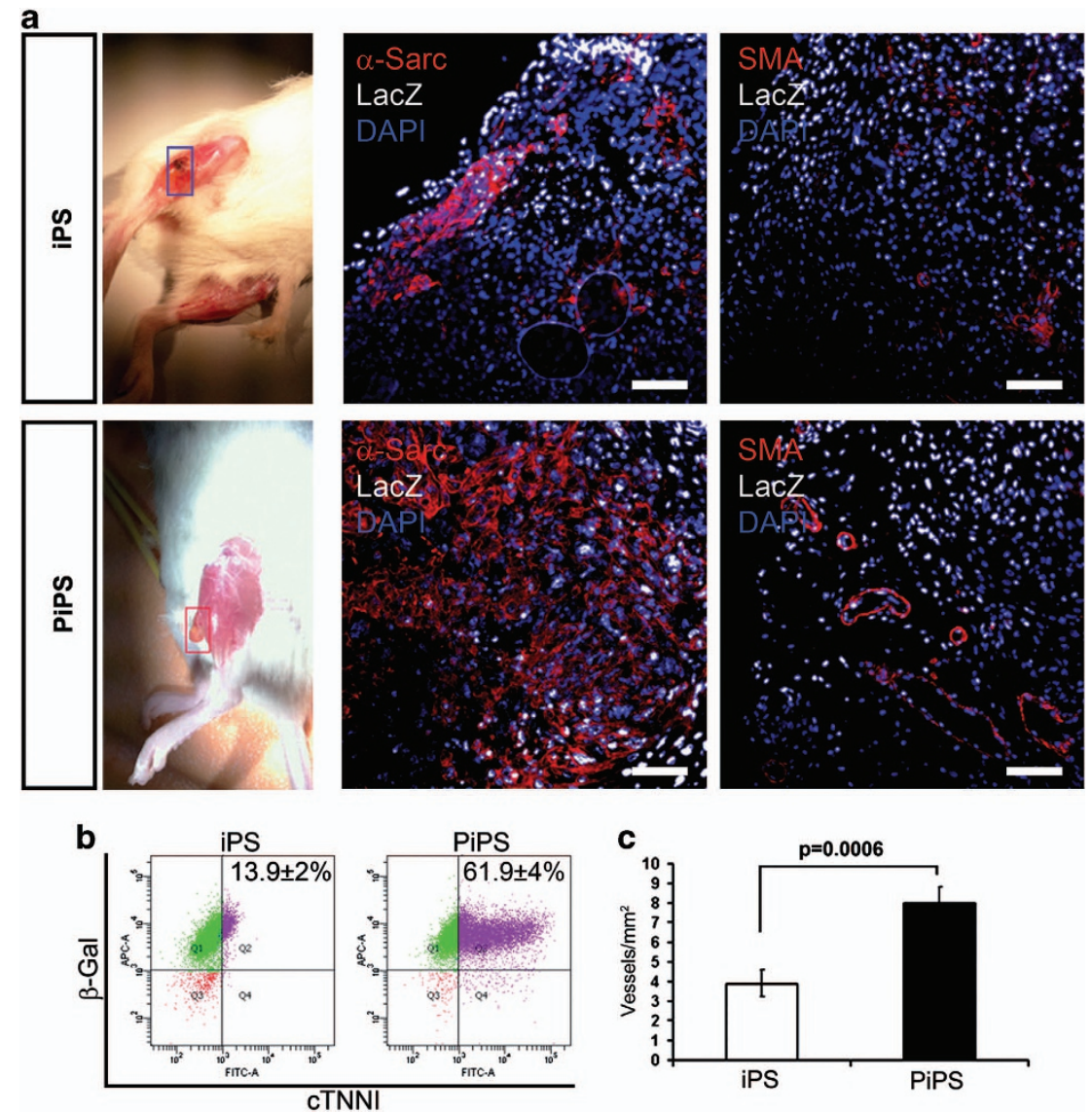

Figure 4 Subcutaneous implantation of PF scaffolds seeded with differentiated, bioengineered iPS cells in mice. (a, left) Extracardiac muscle generated from PEG-fibrinogen $+1 \%$ PEG-diacrylate (PF $+1 \%$ PEG-DA) scaffolds seeded with iPS (upper panel) or PiPS (lower panel) cells after 15 days from implantation in immunodeficient NOD-SCID mice; (a, middle and right) immunofluorescence assays demonstrating the presence of $\alpha$-sarcomeric actin ( $\alpha$-Sarc), indicative of the presence of cardiomyocytes, and $\alpha$-smooth muscle actin (SMA), indicative of the presence of vessels, inside the neo-formed tissue. The exogenous origin of the cardiomyocytes and vessels was proven by nuclear expression of $\mathrm{nLacZ}$. The nuclei were stained with DAPI. White bars $=100 \mu \mathrm{m}$. (b) FACS analysis scatter plots presenting the percentages of $\beta$-galactosidase ( $\beta$-Gal) and cardiac troponin I (cTNNI) double-labeled cells isolated from the newly formed structures by digestion. Mean \pm S.E.M.; $N=4$. (c) Graph of vessel density within the tissue formed by iPS or PiPS cells. Mean \pm S.E.M.; $N=4$

the velocity of blood flow in the left ventricular outflow tract in the designated time span - was nearly restored to physiologic values after 1 month in the MiPS + PiPS cell-scaffold group. Detailed analysis of the results revealed that the biomaterial seeded with both engineered cell types was the only that produced a marked improvement after 30 days of administration (Supplementary Figure 3 online). A representative infarcted mouse heart with a regenerated area (rectangle) is given in Figure $5 \mathrm{~d}$.

Taken together, these results underscore the efficacy of using a combined approach - that is, implantation of a biomaterial seeded with bioengineered cells - for the generation of functional cardiac tissue to prevent adverse cardiac remodeling.

\section{Discussion}

We report here for the first time an approach for the in vivo cardiogenic differentiation of PF-hydrogel-encapsulated iPS cells. Our data demonstrate that a PEGylated fibrinogen scaffold, optimized for stiffness through the use of additional
PEG-DA crosslinker, promoted and accelerated cardiac differentiation of iPS cells. In addition, graft vascularization was enhanced by engineering iPS cells to produce PIGF, whereas the secretion of MMP9 improved engraftment by decreasing scar formation. This combination increased the survival of engrafted cells when injected into the heart of a mouse model of myocardial infarction, and led to less ischemic damage. In fact, \%FS and systolic and diastolic functions were significantly improved in comparison with the other treatment conditions tested in this study.

Several studies and preclinical surveys have reported reduced graft retention - and hence poor and unsatisfactory recovery - because of a very low survival rate of therapeutic cells when these are used alone., ${ }^{1,2,21}$ Increasing emphasis has therefore been placed on the use of biomaterials in combination with cells as a means of improving graft retention and survival. Recent attempts at restoring cardiac tissue function have used such combinations in addition to modulation of the biomaterial features in order to design cell-delivery systems that are biocompatible, biofunctional, bioactive, and reabsorbable, ensuring optimal cell-matrix interactions and 

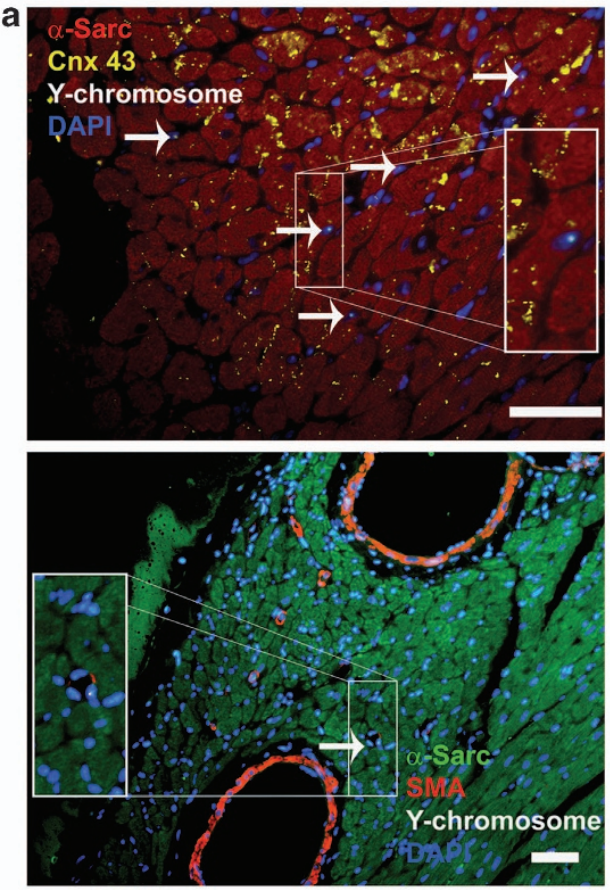

C

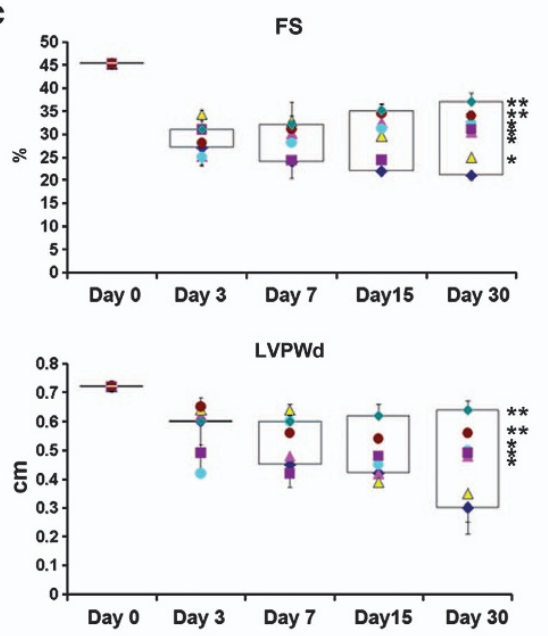

b
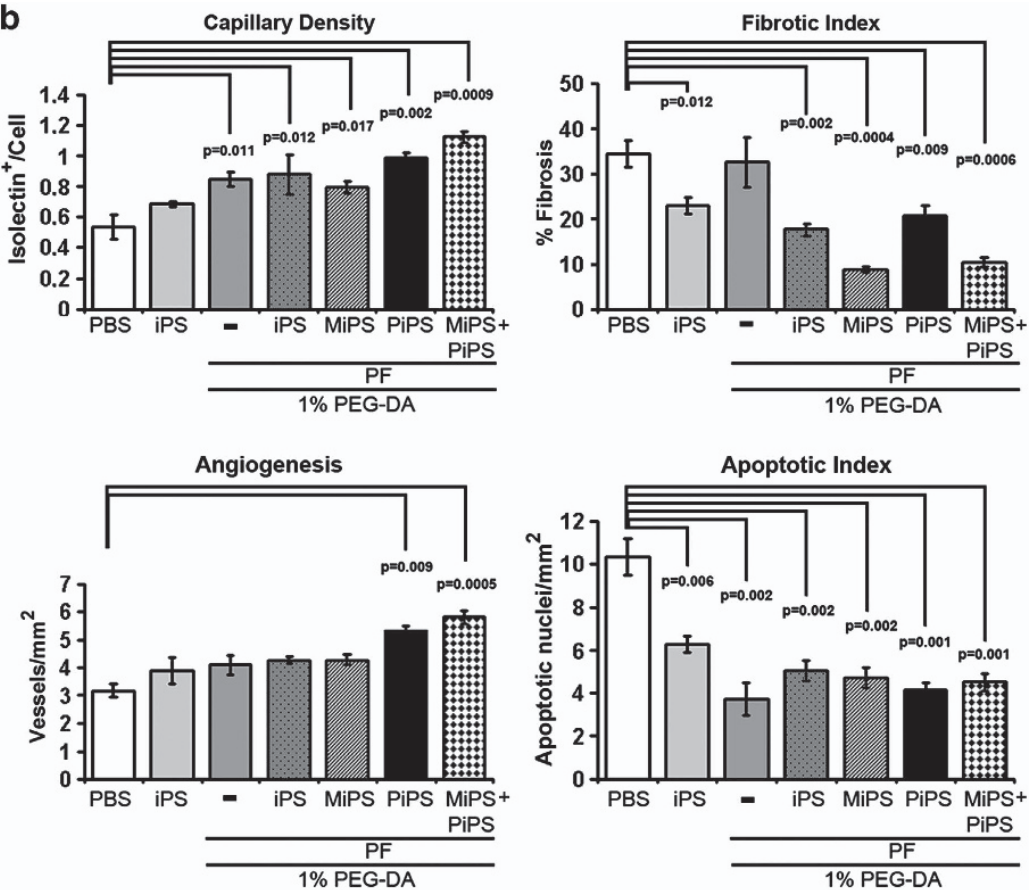

d

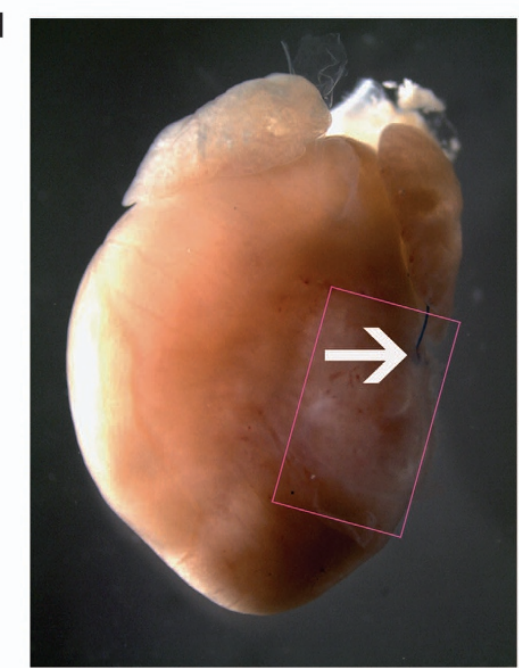

Figure 5 Cardiac implantation of PF scaffolds seeded with differentiated, bioengineered iPS cells in infarcted mice. (a, upper) Representative immunofluorescence image demonstrating the exogenous origin, that is, Y-chromosome positivity (white), of newly formed, $\alpha$-sarcomeric actin-positive cardiomyocytes ( $\alpha$-Sarc, red) in a female ischemic heart injected with PF $+1 \%$ PEG-DA seeded with both MiPS and PiPS cells deriving from a male mouse. Connexin 43 (CNX 43) expression in yellow; (a, lower) immunofluorescence image of an exogenous ( $Y$ chromosome positive) cell (white) contributing to the formation of $\alpha$-smooth muscle actin-positive vessel (SMA, red) in female ischemic cardiac tissue ( $\alpha$-Sarc, green) injected with PF $+1 \%$ PEG-DA scaffold seeded with both MiPS and PiPS cells derived from a male mouse. Nuclei were stained with DAPI (blue). In both images, arrows indicate some of the positive cells. White bars $=100 \mu \mathrm{m}$; higher magnification views are given in the rectangles. (b) Histological analysis of capillary density, fibrotic index, angiogenesis, and apoptotic index. IPS, MiPS, PiPS, and MiPS plus PiPS cells were encapsulated in PEG-fibrinogen (PF) supplemented with an additional 1\% PEG-DA and injected into ischemic myocardium after coronary ligature. Control groups were injected with iPS cells resuspended in PBS, with PBS only, or with PF only. (c) Echocardiographic analysis: percent fractional shortening (\%FS), left ventricular posterior wall diameter in diastole (LV PWd), and left ventricular velocity/ time integral (LV VTI). Effects of IPS, MiPS, PiPS, and MiPS plus PiPS cells seeded onto PF $+1 \%$ PEG-DA and injected after coronary occlusion; control groups received PBS, iPS cells resuspended in PBS, or PF without cells. Boxes represent 95 th percentile; ${ }^{*} P<0.05,{ }^{* *} P<0.01$ versus PBS. Mean $\pm S$.E.M.; $N=4$ (b and c). (d) Representative image of an ex vivo infarcted female heart injected with male MiPS and PiPS cells supported on a PF $+1 \%$ PEG-DA scaffold at 30 days after left coronary artery ligature (arrow)

cell signaling. ${ }^{22-25}$ Fundamentally, this approach entails recreating the most accurate environment mimicking the cardiac ECM. The structural molecular signals, and the biofeedback associated with the cell-biomaterial interface, must work together to drive the transplanted cells toward a desired and stable phenotype. The control of cell behavior with the use of biomaterials was addressed recently through the presentation of immobilized molecules and cellular signals 
from the substrate, as well as mechanically stressed cellular environments. ${ }^{26-28}$

Using the PF hydrogel properties, including optimization of its stiffness, we designed and investigated a 3D encapsulating environment that can be used for cultivating CMs derived from iPS cells. Unlike naturally derived fibrinogen hydrogels, a combination of PEG and fibrinogen was found to improve the mechanical stability of the scaffold so as to tolerate the high repetitive stresses and proteolytic activity of the ischemic myocardium, and, importantly, to prevent cardiac remodeling and scar expansion.

iPS cell-based strategies hold great potential for future clinical application, but we are still far from translating them to the clinic. More basic research needs to be undertaken, for instance to find the most appropriate stage of differentiation for grafting iPS cells. In fact, because iPS cells pass through multiple stages of differentiation before becoming functionally mature CMs, implanting iPS cell-derived cells that are too immature may risk generating cells that are not the required type or that develop into tumors. On the other hand, if engrafted when too mature, they might not be adaptable to the surrounding environment and, hence, conducive to improving cardiac function. Moreover, although the prospective of using cardiomyocyte-derived iPS cells for clinical applications is negligible, they do provide an optimal system with which to study and develop myocardial repair strategies.

\section{Materials and Methods \\ Lentiviral transduction. Cardiac-specific lentiviral vectors carrying Oct4, Sox2, KIf4 (OSK), nLacZ, PIGF, or MMP9 were generated with a standard procedure. ${ }^{5,11}$ Neonatal mouse cardiomyocytes were isolated from 1-day-old mice and reprogrammed with OSK to generate iPS cells as previously reported. ${ }^{5}$ iPS cells were transduced with lentiviral vector carrying $n L a c Z$ and used as a control, or with those carrying PIGF or MMP9 to generate PIGF- and MMP9-secreting cells (respectively termed PiPS and MiPS cells). iPS cells were then redifferentiated into cardiomyocytes by exposure to $2.5 \mathrm{ng} / \mu \mathrm{l}$ BMP2 and induction of EB formation via the hanging-drop culture method. ${ }^{5}$}

Biomaterial preparation. PEG-fibrinogen (PF) and the PEG-DA crosslinker were prepared as described previously. ${ }^{18,29,30}$ Briefly, PF was prepared at a desired concentration and diluted with sterile PBS as required. To increase the stiffness of the scaffold, an additional 1 or 2\% PEG-DA was added to the basic PF mixture in some cases. The photoinitiator Irgacure 2959 (Ciba Specialty Chemicals, Basel, Switzerland) was added to the PF at a final concentration of $0.1 \% \mathrm{w} / \mathrm{v}$. Cells were added at the desired concentration and the solution immediately exposed to UV light to initiate polymerization within glass tubes.

Invasion and angiogenic assays. The Boyden chamber assay was used to assess the migratory potential of cells when stimulated with either $100 \mathrm{ng} / \mathrm{ml}$ SDF-1 or $100 \mathrm{ng} / \mathrm{ml} \mathrm{SCF}$. In vitro capillary formation was assessed in HUVECs stimulated with conditioned media from iPS cell cultures. QRT-PCR, histology, FACS analysis, immunofluorescence microscopy, and echocardiography were all done using standard techniques.

Subcutaneous implantation of constructs in mice. Cylindrically shaped PF plugs encapsulating either $1 \times 10^{6}$ iPS or PiPS cells and polymerized in vitro were implanted underneath the skin of NOD-SCID mice at the level of the TA muscle. Mice were killed 2 weeks after the implantation, and growths found on the TA muscle collected.

AMI mouse model and intrainfarct implantation of constructs. The left descending coronary artery of female NOD-SCID mice was surgically ligated in order to produce an ischemic area in the left ventricle. iPS, MiPS, or PiPS $\left(5 \times 10^{5}\right)$ cells, all generated from male mice, were resuspended separately or in combination with hydrogels just before use and injected into the ischemic area right after the ligation. The combinations were: IPS cells encapsulated in PF supplemented with an additional 1\% PEG-DA (PF $+1 \%$ PEG-DA); MiPS cells encapsulated in PF + $1 \%$ PEG-DA; PiPS cells encapsulated in PF + 1\% PEG-DA; and MiPS + PiPS cells in PF + 1\% PEG-DA. PF without iPS cells was injected either into the border zone or into the infarcted area to evaluate the supporting functions of the scaffold after the ischemic event in a control group. The presence of functional tissue together with its effects on cardiac function was evaluated by immunofluorescence, histological assays, and echocardiography.

Statistical analysis. Linear-model two-way repeated measures ANOVA was used to confirm the differences in over-time-dependent surveys. One-way ANOVA was used to evaluate the variations in percentage of regenerated areas and grafted cells between experimental groups and the control. All results are expressed as mean \pm S.E.M. Student's $t$-test was used to confirm the differences between the best proposed approach and the other groups.

\section{Conflict of Interest}

The authors declare no conflict of interest.

Acknowledgements. This work was supported by the Italian Ministry of Health - Giovani Ricercatori 2009 (GR-2009-1606636); by the European Community - 7th Framework Programme (NMP3-LA-2008-214402 ANGIOSCAFF); and by the Super Pig Programme co-financed by the Lombardy Region (Institutional Agreements no. 14388A).

1. Pal SN, Kofidis T. New cell therapies in cardiology. Expert Rev Cardiovasc Ther 2012; 10: 1023-1037.

2. Laflamme MA, Murry CE. Heart regeneration. Nature 2011; 473: 326-335.

3. Takahashi K, Yamanaka S. Induction of pluripotent stem cells from mouse embryonic and adult fibroblast cultures by defined factors. Cell 2006; 126: 663-676.

4. Takahashi K, Tanabe K, Ohnuki M, Narita M, Ichisaka T, Tomoda K et al. Induction of pluripotent stem cells from adult human fibroblasts by defined factors. Cell 2007; 131: 861-872.

5. Rizzi R, Di Pasquale E, Portararo P, Papait R, Cattaneo P, Latronico MV et al. Post-natal cardiomyocytes can generate iPS cells with an enhanced capacity toward cardiomyogenic re-differentation. Cell Death Differ 2012; 19: 1162-1174.

6. Diez Villanueva P, Sanz-Ruiz R, Nunez Garcia A, Fernandez Santos ME, Sanchez PL, Fernandez-Aviles F. Functional multipotency of stem cells: what do we need from them in the heart? Stem Cells Int 2012; 2012: 817364.

7. De Falco S, Gigante B, Persico MG. Structure and function of placental growth factor. Trends Cardiovasc Med 2002; 12: 241-246.

8. De Falco S. The discovery of placenta growth factor and its biological activity. Exp Mol Med 2012; 44: 1-9.

9. Van den Steen PE, Dubois B, Nelissen I, Rudd PM, Dwek RA, Opdenakker G. Biochemistry and molecular biology of gelatinase B or matrix metalloproteinase- 9 (MMP-9). Crit Rev Biochem Mol Biol 2002; 37: 375-536.

10. Fuoco C, Salvatori ML, Biondo A, Shapira-Schweitzer K, Santoleri S, Antonini S et al. Injectable polyethylene glycol-fibrinogen hydrogel adjuvant improves survival and differentiation of transplanted mesoangioblasts in acute and chronic skeletal-muscle degeneration. Skelet Muscle 2012; 2: 24.

11. Gargioli C, Coletta M, De Grandis F, Cannata SM, Cossu G. PIGF-MMP-9-expressing cells restore microcirculation and efficacy of cell therapy in aged dystrophic muscle. Nat Med 2008; 14: 973-978.

12. Iwasaki H, Kawamoto A, Tjwa M, Horii M, Hayashi S, Oyamada A et al. PIGF repairs myocardial ischemia through mechanisms of angiogenesis, cardioprotection and recruitment of myo-angiogenic competent marrow progenitors. PLOS One 2011; 6: e24872.

13. Morgan J, Rouche A, Bausero P, Houssaini A, Gross J, Fiszman MY et al. MMP-9 overexpression improves myogenic cell migration and engraftment. Muscle Nerve 2010; 42: 584-595.

14. Lam MT, Wu JC. Biomaterial applications in cardiovascular tissue repair and regeneration. Expert Rev Cardiovasc Ther 2012; 10: 1039-1049.

15. Liau B, Zhang D, Bursac N. Functional cardiac tissue engineering. Regen Med 2012; 7: 187-206.

16. Seliktar D. Extracellular stimulation in tissue engineering. Ann NY Acad Sci 2005; 1047: 386-394.

17. Seliktar D. Designing cell-compatible hydrogels for biomedical applications. Science 2012; 336: 1124-1128. 
18. Shapira-Schweitzer K, Seliktar D. Matrix stiffness affects spontaneous contraction of cardiomyocytes cultured within a PEGylated fibrinogen biomaterial. Acta Biomater 2007; 3 : 33-41.

19. Sarig-Nadir O, Livnat N, Zajdman R, Shoham S, Seliktar D. Laser photoablation of guidance microchannels into hydrogels directs cell growth in three dimensions. Biophys J 2009; 96: 4743-4752.

20. Puceat M. Protocols for cardiac differentiation of embryonic stem cells. Methods 2008; 45 168-171.

21. Jones DA, Choudry F, Mathur A. Cell therapy in cardiovascular disease: the national society journals present selected research that has driven recent advances in clinical cardiology. Heart 2012; 98: 1626-1631.

22. Segers VF, Lee RT. Biomaterials to enhance stem cell function in the heart. Circ Res 2011; 109: 910-922.

23. Zwi-Dantsis L, Gepstein L. Induced pluripotent stem cells for cardiac repair. Cell Mol Life Sci 2012; 69: 3285-3299.

24. Udelsman BV, Maxfield MW, Breuer CK. Tissue engineering of blood vessels in cardiovascular disease: moving towards clinical translation. Heart 2013; 99: 454-460.

25. Kurobe H, Maxfield MW, Breuer CK, Shinoka T. Concise review: tissue-engineered vascular grafts for cardiac surgery: past, present, and future. Stem Cells Transl Med 2012; 1: $566-571$.
26. Discher DE, Mooney DJ, Zandstra PW. Growth factors, matrices, and forces combine and control stem cells. Science 2009; 324: 1673-1677.

27. Nemir S, West JL. Synthetic materials in the study of cell response to substrate rigidity. Ann Biomed Eng 2010; 38: 2-20.

28. Marklein RA, Burdick JA. Controlling stem cell fate with material design. Adv Mater 2010; 22: $175-189$.

29. Dikovsky D, Bianco-Peled H, Seliktar D. Defining the role of matrix compliance and proteolysis in three-dimensional cell spreading and remodeling. Biophys $\mathrm{J} 2008$; 94 : 2914-2925.

30. Dikovsky D, Bianco-Peled H, Seliktar D. The effect of structural alterations of PEG-fibrinogen hydrogel scaffolds on 3-D cellular morphology and cellular migration. Biomaterials 2006; 27: 1496-1506.

(c) (i) $\odot$ Cell Death and Disease is an open-access journal published by Nature Publishing Group. This work is licensed under a Creative Commons Attribution-NonCommercialNoDerivs 3.0 Unported License. To view a copy of this license, visit http://creativecommons.org/licenses/by-nc-nd/3.0/

Supplementary Information accompanies this paper on Cell Death and Disease website (http://www.nature.com/cddis) 